\title{
ACCULTURATION AND COPING STRATEGIES IN THE WORKPLACE
}

\section{Stoyanka Hristova ${ }^{7}$}

\begin{abstract}
The paper presents empirical study of the level of organizational acculturation and the frequency of use of coping strategies in Bulgarian sample. The relationship between them and their dependence on socio-demographic factors were also examined. The aim of the research conducted was to verify the hypotheses that certain socio-demographic factors had a statistically significant impact on the phenomena studied, and that the use of controloriented coping strategies was related to higher levels of acculturation in organization.

The results obtained when applying analysis of variance indicated statistically significant differences in the level of acculturation and the frequency of use of coping strategies depending on part of the socio-demographic factors observed, confirming partially the first hypothesis formulated.

The second hypothesis was fully confirmed. The findings of correlation analysis indicated that high levels of acculturation in organization correlated statistically significantly with the coping strategies of Increasing efforts, Confidence in success, Change of situation and Time management, all of which are part of the control-oriented coping.
\end{abstract}

KEY WORDS: Acculturation, Acculturative stress, Work stress, Coping strategy, Control-oriented coping

JEL: M14

UDC:005.591:005.21

005.72:005.74(497.2)

COBISS.SR-ID 227948812

${ }^{7}$ Corresponding author, Sofia University “St. Kiment Ohridski”, Bulgaria, e-mail: stoyanka.shopova@gmail.com 


\section{INTRODUCTION}

In recent decades, the incidence of stress and its effects on both a personal and organizational level is increasingly growing due to significant changes in the workplace caused by globalization, cultural diversity of workforce and the increasing need of new and different skills and qualifications. The data and results of numerous scientific studies and research have given grounds for adding new factors and features to the basic components presented in the models of stress from the 80 s of the 20th century. In this regard, Palmer, Cooper and Thomas (Palmer et al.,2004) have considered culture as one of the main categories of potential sources of stress manifested in each of the other categories of stressors (requirements, control, relationships change, role, support). The psychological stress is more often studied and analyzed through the perspective of cultural diversity, intercultural interaction and cross-cultural transition, based on the so-called "medical" and "cognitive" models of stress. While the medical model fully supports the idea of inevitable pathological response as a result of intercultural contact, the cognitive approach recognizes it partly, mostly in terms of transition and change that are considered as stressful events and highlights the coping process and successful adaptation to the new environment. It involves social aspects of the adaptation process and focuses not only on the individual but also on the wider socio-cultural environment. Adaptation is realized in different ways, one of which is the one-way adjustment to difficult or stressful situation. And the degree of success of the adaptation depends not only on personal factors, as postulated in the clinical approach, but also on a number of social and cultural factors (Ward et al., 2003). That more complex view of the relationship between cross-cultural transition and stress is reflected in the work of Berry (Berry, 2001, 2002, 2006a, 2006b, 2006c) on issues related to the process of acculturation and acculturative stress.

\section{THEORETICAL BACKGROUND}

The acculturation theory reflects the problematic effects arising from intercultural contact, through the phenomena of adaptation and stress. Acculturation is most often defined as a change in two or more cultural systems arising as a result of the two-way diffusion of culture elements. It indicates the relationship between cultural differences and the process of cultural change and the intergroup adaptation, especially in the case of merger and acquisition of one or more minority groups by the majority (Berry, 2001). The process is carried out both on group and individual level and passes through three main stages: contact between at least two groups, leading to a certain modification; conflict caused by the dominance of one group over the other / others, and adaptation (Berry, 2001). According to Berry as a result of interaction with two or more cultures, the individual passes through at least two levels of change. On the first level occur behavioral changes that affect the behavior pattern of the individual in situations characterized by cultural diversity in terms of speech patterns, customs, nutrition, style of dress, and even selfidentity. The second level covers the process of acculturative stress that includes emotional reactions such as anxiety and depression by the individual (Berry, 2006b, 2006c).

Acculturative stress is generally defined as moderately pathological and destructive individual states and behaviors resulting from contact with another cultural group. Its symptoms include anxiety, depression, confusion, low self-esteem, psychosomatic diseases (Berry, 2006c). Berry has offered a model based on stress-coping approach that included coping strategies and resources as moderating factors on individual level occurring during acculturation, and socio-demographic factors (such as, age, gender, education, religion, 
health, language, status) defined as moderating factors on individual level that have occurred prior to acculturation (Berry, 2006b).

Coping strategies are widely discussed in the scientific and professional literature on stress. Here we will focus briefly on the theoretical formulation of Latack, according to which coping is a dynamic process and it involves cognitive appraisals of perceived stressor, of its characteristics and requirements for performing certain actions, of the resources needed to carry out those actions, and of their consequences. It manifests in reducing the external and internal requirements causing stress by handling the situation perceived as dangerous, challenging or threatening, as the choice of a coping strategy is determined by the environment variables and personality. Latack has outlined three main categories of coping strategies related to occupational stress:

- Strategies of control covering proactive and confronting the stress actions and cognitive reappraisals;

- Avoidance strategies, which include actions and cognitive reappraisals aimed at avoiding the problem;

- Strategies of managing the stress symptoms involving other widely accepted ways of dealing with the symptoms of occupational stress as relaxation, exercise and more (Latack, Havlovic, 1992).

\section{METHODOLOGY}

Since coping strategies are indicated as moderating factor in Berry's model of acculturative stress, any empirical data showing the presence or absence of relationship between acculturation and coping in the workplace would be of interest. In this regard, the main objective of this study was to establish empirically the specific characteristics of acculturation and coping strategies in contemporary organizations, and the relation between them both.

In the hypotheses formulated it was assumed that:

1. The socio-demographic factors: age, marital status, level of education, total work experience, work experience in the particular organization, position in the organizational hierarchy, have a statistically significant impact on the level of organizational acculturation and the frequency of use of coping strategies.

2. The use of control-oriented coping strategies is related to higher levels of acculturation in organization.

The main tasks in response to the needs of the empirical study, were:

1. To define the mean values of the variables studied.

2. To find statistically significant differences in the level of organizational acculturation and the frequency of use of coping strategies, depending on the following socio-demographic factors: age, marital status, level of education, total work experience, work experience in the particular organization, position in the organizational hierarchy.

3. To find out if there were statistically significant correlations between organizational acculturation and the coping strategies used. 
The research tools applied for the purposes of this empirical study included:

1. Organizational Acculturation Scale - subscale of the Index of Organizational Assimilation (Gailliard et al., 2010). It includes 5 items related to the acceptance and utilization of organizational norms and standards. According to the factor analysis applied in this study, there is one general factor outlined covering all items and explaining $63.79 \%$ of the total variance. Psychometric properties of the scale were very good - Cronbach's alpha coefficient was 0.86 (for comparison, reliability of Organizational Acculturation Scale indicated by its authors was $\alpha=0.84$ ).

2. Coping Strategies Questionnaire (Latack, 1986) - standardized for Bulgarian conditions by Ilieva (Ilieva, 2008 ). The questionnaire examines preferences and frequency of use of certain actions and cognitive appraisals aimed at avoiding a stressful situation (escape-oriented coping strategies) or exercising control over it (control-oriented coping strategies). It consists of 28 items divided into seven factors: Increase of efforts, Escape from situation, Confidence in success, Seeking social support, Change of situation, Time management, Reconciliation with situation which, as a result of a factor analysis conducted in this study, explain $63.54 \%$ of the total variance. In this sample, the questionnaire showed good psychometric properties $(\alpha=0.74)$.

\section{Subject of study:}

In the empirical study participated 219 respondents, $26.5 \%(\mathrm{~N}=58)$ of which were representatives of the male gender, and $73.5 \%(\mathrm{~N}=161)$ were women. The age range of the sample was between 18 and 67 years of age $(\mathrm{M}=34.32)$, as the most numerous was the group of participants aged up to 29 years $(40.2 \% ; \mathrm{N}=88), 31.9 \%(\mathrm{~N}=70)$ of the participants were of 30 to 39 years of age, $16.9 \%(\mathrm{~N}=37)$ of the participants were aged from 40 to 49 years, and $11 \%(\mathrm{~N}=24)$ of the participants were aged above 49 years.

\section{ANALYSIS AND INTERPRETATION OF STUDY RESULTS}

In order to be verified the hypotheses raised, were conducted descriptive analysis, analysis of variance and correlation analysis of empirical data from this study.

The descriptive analysis outlined relatively high levels of organizational acculturation $(\mathrm{M}=19.8 ; \mathrm{SD}=3.9)$, indicating the good understanding, perception and utilization of organizational cultural values and practices. The majority of respondents chose the most effective and minimizing acculturative stress strategy to adapt to the values and practices of the organization in which they work, which further facilitates the reduce of the levels of work stress and its retention in the moderate range.

The mean values in terms of coping strategies show that in the event of a stressful situation at work, the most frequently used coping strategy for this sample was the Increase of efforts $(\mathrm{M}=24.42 ; \mathrm{SD}=3.93)$, which is related to making actions and cognitive reappraisals, improving the performance of working duties. The second most preferred strategy for coping with work stress was the Confidence in success $(\mathrm{M}=18.92$; $\mathrm{SD}=3.87$ ), which includes a positive perception of stressful situations as an opportunity to acquire new skills and experience. At the third place by preferences and frequency of use, was ranked the pursuit of Change of situation $(\mathrm{M}=16.29$; $\mathrm{SD}=3.82)$, followed by the strategies associated with Seeking social support $(\mathrm{M}=13.1 ; \mathrm{SD}=2.68)$ and Time management $(\mathrm{M}=7.93 ; \mathrm{SD}=1.41)$. At the penultimate place was the strategy aimed at 
Escape from situation $(\mathrm{M}=6.84 ; \mathrm{SD}=2.06)$. Reconciliation with situation $(\mathrm{M}=5.94 ; \mathrm{SD}$ $=1.68$ ) was the least preferred coping strategy.

Overall, in this sample dominated the attitude towards using strategies typical for the active control-oriented coping, identified in the scientific literature as the most effective and suitable way to cope with stress factors.

As a result of the analysis of variance conducted, were found statistically significant differences both in the level of organizational acculturation and the frequency of use of certain coping strategies depending on the following socio-demographic factors: age, marital status, level of education, total work experience, work experience in the particular organization, position in the organizational hierarchy.

\section{Impact of socio-demographic factors on the levels of organizational acculturation}

In analyzing the impact of socio-demographic factors on the level of organizational acculturation were wound statistically significant differences (at $\mathrm{p}<0.01$ ) depending on age $(F=4.142 ; p=0.007)$, work experience in the particular organization $(F=5.297 ; p=$ $0.002)$, and position in the organizational hierarchy $(F=5.463 ; p=0.005)$, occupied by the participants in this study.

In terms of the Age factor, there was a gradual increase in the mean values of the variable together with the increase in the age of respondents - those who were over 49 years old acculturated to the organization in which they work to the greatest extent $(M=21.15$; $\mathrm{SD}=3.1$ ), as opposed to the group of the youngest participants of age up to 29 years, in which the degree of organizational acculturation was lowest $(\mathrm{N}=19.03$; $\mathrm{SD}=3.5)$. The difference between the mean values in both groups was 2.12 (at the level of $\mathrm{p}<0.05$ ) (Fig. 1). The possible explanation here is associated with the typical for maturity reassertion and improvement of qualities such as tolerance, respect, recognition and evaluation not only of "one's own", but also of "others'" norms and values, and building skills of faster and more effective adjustment to them.

The results were similar in terms of work experience in the particular organization. The degree of organizational acculturation among persons which had less than 2 year work experience in the present organization $(M=18.73$; $S D=3.94)$, gradually increased with 3.33 units and achieved mean value $\mathrm{M}=22.06$ (with standard deviation $\mathrm{SD}=2.41$ ) in participants which had more than 10 year work experience in the specific organization (Fig. 1). Tukey HSD test indicated the presence of statistically significant differences, except between those two groups (at the level of $\mathrm{p}<0.01$ ), also between the group of persons with less than 2 year work experience in the specific organization and the group of people with work experience from 6 to 10 years in the organizations in which they work at present, as the difference between the mean values of both groups is 1.99 points (at the level of $\mathrm{p}$ $<0.05$ ). Statistically significant difference of 2.4 points (at the level of $p<0.05$ ) was found also between the group of participants with 2 to 5 years work experience and those with over 10 years work experience in the particular organization (Fig. 1). Apparently, a longer period of work in a certain organization allows for more detailed and substantial awareness with both formal and informal organizational norms, values, practices and standards which promotes their adoption and utilization by individuals. 


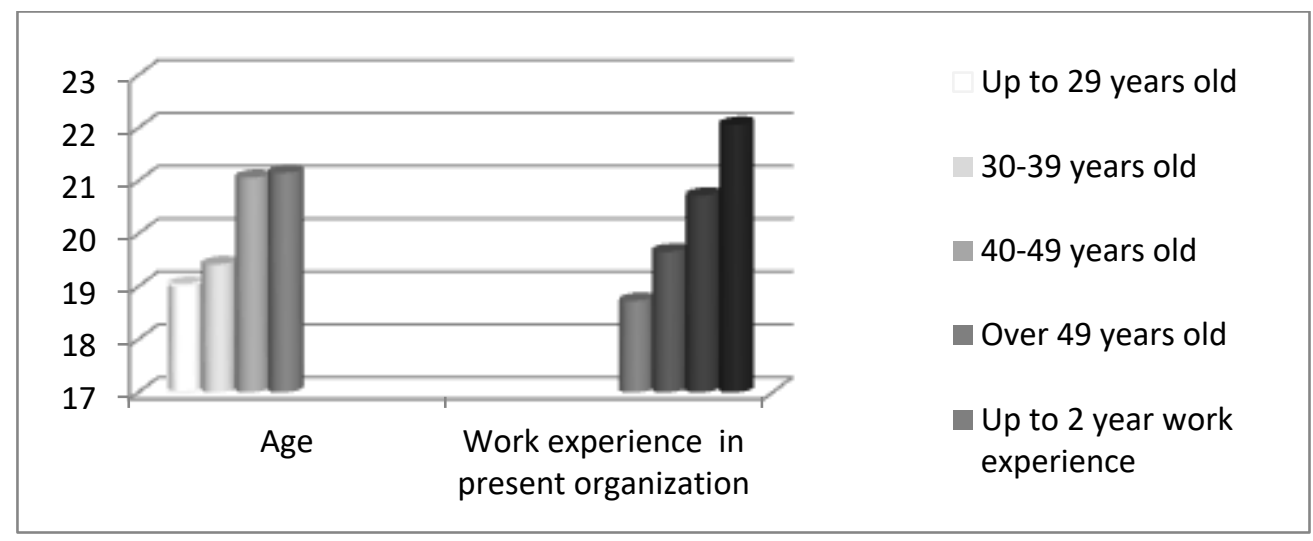

Figure 1: Level of Organizational acculturation depending on Age

and Work experience in present organization $(p<0.01)$

Another socio-demographic factor that led to statistically significant differences in the degree of organizational acculturation between groups was the position in the organizational hierarchy occupied by respondents. The group of employees and workers having only executive functions $(\mathrm{M}=18.74$; $\mathrm{SD}=3.7)$ was characterized with the lowest mean values of this variable, and the highest values were observed in the group of persons holding managerial positions $(\mathrm{M}=21.00 ; \mathrm{SD}=2.79)$, as the difference between them was 2.26 points (at the level of $\mathrm{p}<0.01$ ) (Fig. 2). High ambitions and strong motivation for success and career development typical for people occupying managerial positions, were likely the reason for better understanding and adapting to organizational culture.

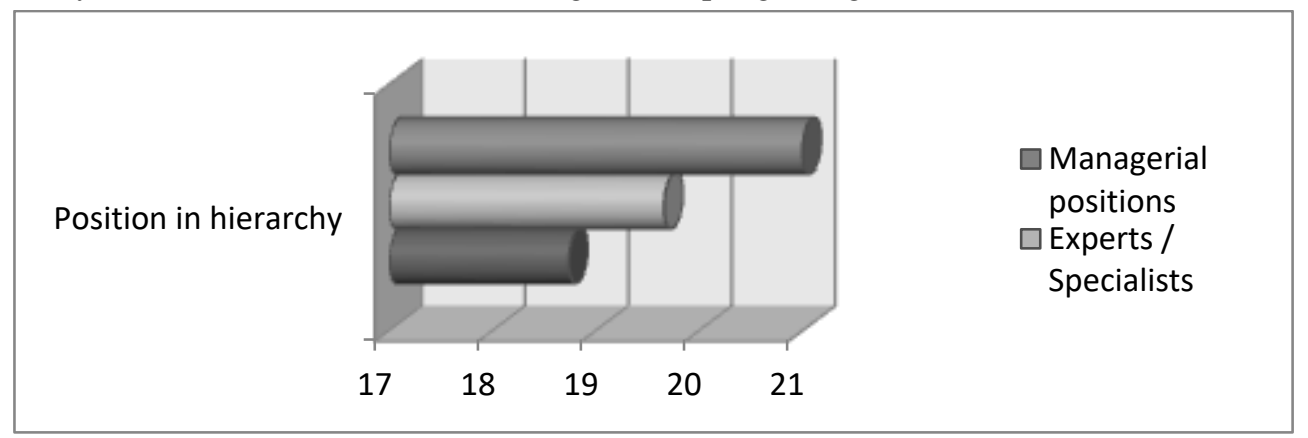

Figure 2: Level of Organizational acculturation depending on

Position in organizational hierarchy $(p<0.01)$

In summary, higher levels of organizational acculturation characterized persons which are over 49 years old, having more than 10 year work experience in the specific organization, and holding managerial position in the organizational hierarchy. Conversely, with the lowest level of organizational acculturation were participants aged up to 29 years, having less than 2 year work experience in the particular organization, and occupying the lowest employee / worker positions in the organizational hierarchy. The observed classic confrontation of maturity and youth, of experience and inexperience, of management and employees, in this case was in favor of the former and to the detriment of the latter, in terms of socialization and integration in the organization. 


\section{Impact of socio-demographic factors on the frequency of use of coping strategies}

Similar to organizational acculturation, the frequency of use of coping strategies was also influenced to a relatively large degree by certain socio-demographic factors, namely age, level of education, marital status, occupied position in the organizational hierarchy and the total work experience of respondents.

Statistically significant differences (at $\mathrm{p}<0.05)$ depending on age were established in relation to the Time Management coping strategy $(\mathrm{F}=2.877 ; \mathrm{p}=0.038)$, as the frequency of its use graded over the years - it was mostly preferred by participants aged over 49 years $(\mathrm{M}=8.35 ; \mathrm{SD}=1.18)$, and less chosen by people aged up to 29 years $(\mathrm{M}=7.53 ; \mathrm{SD}=$ 1.47), as the difference between the mean values of both groups was 0.82 points (Fig. 3). It turns out that with time passing and the transition from youth to adulthood, the pursuit of effective planning and structuring of working time, particularly in situations of tension and stress, increases.

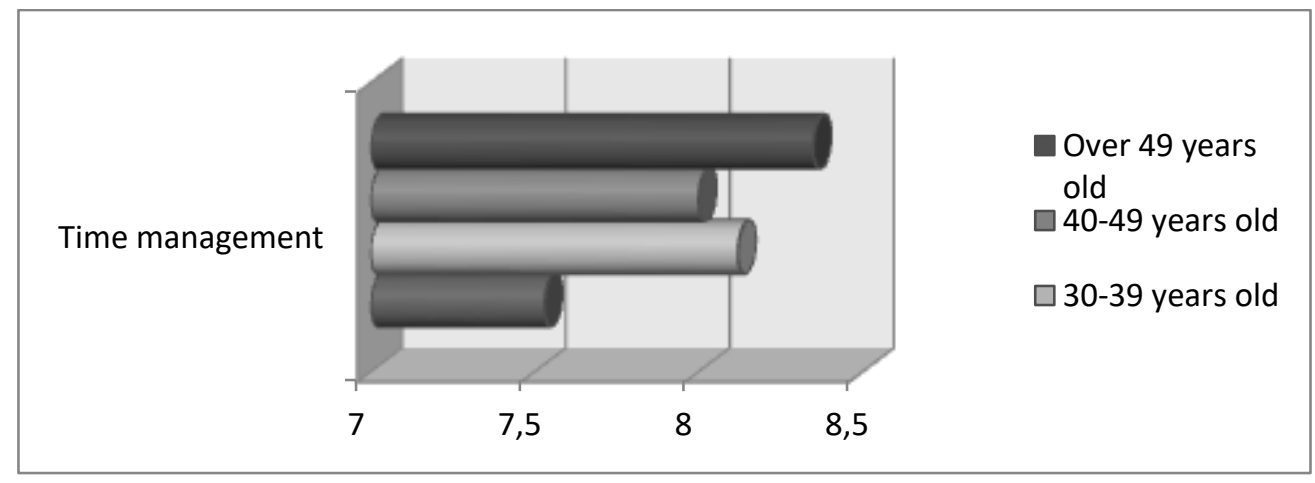

Figure 3:Frequency of use of Time management coping strategy

depending on Age ( $p<0.05)$.

Significant influence on the choice of Time Management as a strategy for coping with stress in the workplace was caused also by the level of education $(F=3.014 ; p=0.031)$ at $\mathrm{p}<0.05$. Here again was observed increase in the frequency of use of this coping strategy in a direct ratio to the raising of the acquired qualification degree - it was mostly chosen by respondents with educational degree greater than higher education $(\mathrm{M}=8.64$; $\mathrm{SD}=1.29)$, and less used by persons who have graduated only primary or secondary school $(\mathrm{M}=7.53$; $\mathrm{SD}=1.65)$, as the difference of mean values between them was 1.11 points. Another coping strategy influenced by the level of education (at $\mathrm{p}<0.01$ ) was the Increase of efforts $(\mathrm{F}=4.119 ; \mathrm{p}=0.007)$ - the highest mean values here were reported by respondents which have acquired educational degree above master's degree $(\mathrm{M}=26.46 ; \mathrm{SD}=3.44)$, and the lowest ones, with a difference of 3.79 points, were found in the group of people with bachelor's degree $(\mathrm{M}=22.67 ; \mathrm{SD}=4.13)$.

Therefore, for those who have continued their education after obtaining a master's degree, there was an affinity for some of the most effective coping strategies - Increasing efforts and Time management, in order to overcome the difficulties or problems in the workplace. In this case, one should not underestimate the role of the experience gained in many stressful and problematic situations during education itself. 
Depending on marital status were found statistically significant differences in the following coping strategies: Change of situation $(\mathrm{t}=2.433 ; \mathrm{p}=0.016)$, at the level of $\mathrm{p}<0.05$, and Time Management $(\mathrm{t}=3.241 ; \mathrm{p}=0.001)$ at the level of $\mathrm{p}<0.01$. In both cases higher values(with a difference of, respectively, 0.40 points and 0.74 points) were reported in the group of married people (respectively, $\mathrm{M}=3.51$; $\mathrm{SD}=1.01$ and $\mathrm{M}=8.12 ; \mathrm{SD}=$ 1.28). Perhaps the sharing of personal life with intimate partner was a prerequisite for developing a greater degree of responsibility, good organizational skills, and active attitude to problem solving - qualities that have significant influence on the choice of coping strategies.

Another factor influencing the frequency of choice of Time management as a strategy for coping with work stress was the total work experience of respondents $(F=3.103 ; p=$ 0.028 ) at the level of $p<0.05$. In accordance with the aforementioned upward gradation of the use of this coping strategy in parallel with age increase, here was also found a tendency of enhancing preferences for it together with the growing of total work experience. Participants with total work experience of over 20 years $(M=8.35$; $S D=1.12)$, reported the highest levels compared to participants with total work experience up to 5 years $(\mathrm{M}=$ $7.62 ; \mathrm{SD}=1.52$ ), as the difference between the mean values of both groups was 0.73 points. Hence, a prerequisite for the frequent choice of this strategy was not just entering in one's more mature years, but also the work experience and habits accumulated over the years (Fig. 4).

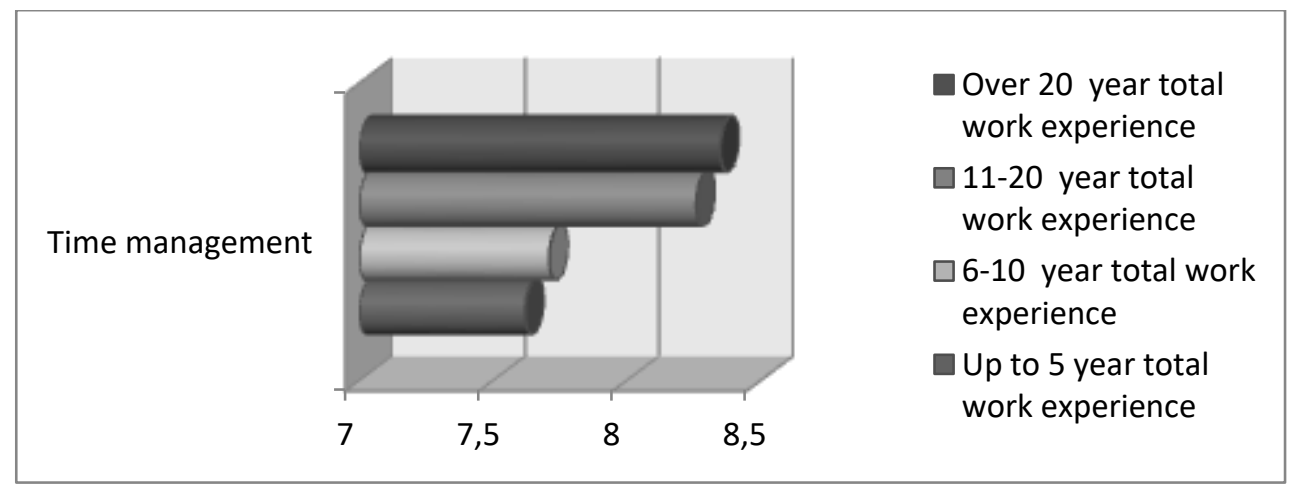

Figure 4:Frequency of use of Time management coping strategy

depending on Total work experience ( $p<0.05)$.

The Escape from problematic situations, pointed in the literature as insufficiently effective coping strategy, was significantly influenced (at $\mathrm{p}<0.05)$ by the position in organizational hierarchy occupied by respondents $(F=3.250 ; p=0.041)$. The highest mean values in this subscale were reported in the group of employees or workers having only executive functions in the organization $(\mathrm{M}=17.11$; $\mathrm{SD}=3.56)$, while the lowest ones were reported in the group of experts and professionals $(M=15.50 ; \mathrm{SD}=3.58)$, with a difference of 1.61 points, according to the Tukey HSD test. Despite expectations for the lowest degree of preference to this strategy by persons with managerial positions, actually the respondents with expert positions were those who have most rarely realized some form of escape from problematic work situations. Therefore, these results are of interest and indicate a need for more thorough analysis and special attention to these issues, as they may be a signal of ineffective stress management by leading cadres (Fig. 5). 


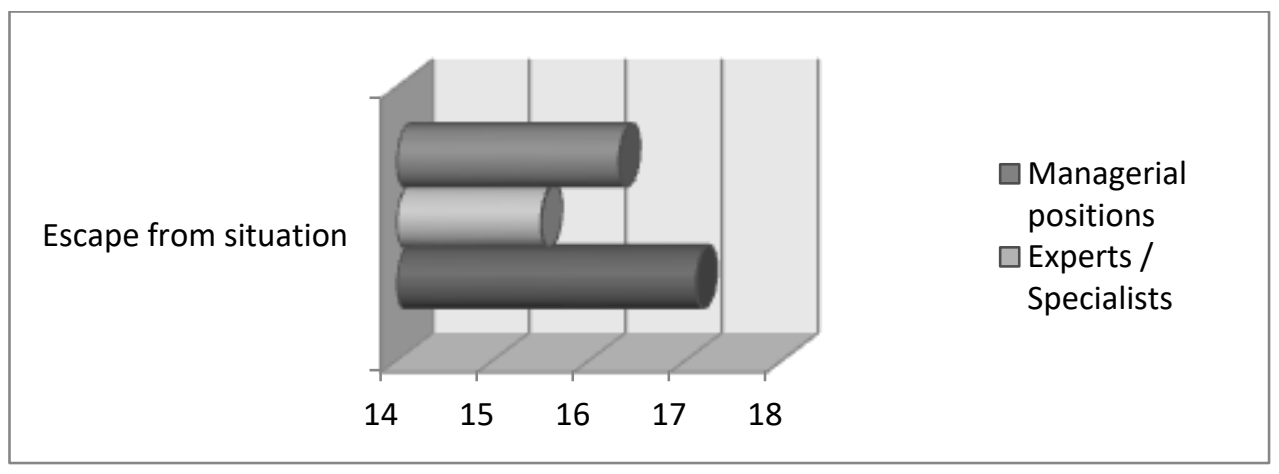

Figure 5: Frequency of use of Escape from situation coping strategy

depending on Position in organizational hierarchy $(p<0.05)$.

It can be summarized that there was statistically significant impact of sociodemographic factors on four of the coping strategies studied, namely: Time Management, Increase of efforts, Change of situation and Escape from situation. Unlike the strategies of Increasing efforts, Changing situations and Escaping from situations, where each of them was influenced by only one socio-demographic factor (respectively, level of education, marital status and position in organizational hierarchy), the Time Management strategy was impacted by the largest number of socio-demographic factors (age, level of education, marital status and total work experience). In this regard, it was most frequently used by respondents which are over 49 years old, with education level higher than master's degree, having husband / wife and more than 20 year total work experience. As opposed to them, the strategy of Time management was least chosen by unmarried people aged up to 29 years, with education level not higher than secondary school, having less than 5 year total work experience. Again, there was a contrast between maturity and youth, experience and inexperience, higher and lower education, as in this case it was in favor of the former in terms of effective coping and stress management in the workplace.

In the correlation analysis of relationships between the phenomena studied, were found three statistically significant correlations (at $\mathrm{p}<0.01)$ of organizational acculturation with coping strategies of Increasing efforts $(\mathrm{r}=0.284 ; \mathrm{p}=0.000)$, Change of situation $(\mathrm{r}=$ $0.198 ; \mathrm{p}=0.004)$ and Time management $(\mathrm{r}=0.178 ; \mathrm{p}=0.009)$, as well as a correlation at the level of $\mathrm{p}<0.05$ between organizational acculturation and the Confidence in success coping strategy $(\mathrm{r}=0.176 ; \mathrm{p}=0.010)$ (Table 1$)$.

All the four correlations were positive and showed relationships in a direct ratio between the variables. With the highest value was the correlation between the degree of acculturation to the organizational culture and coping strategy of Increasing efforts, indicating that frequent taking of actions for increasing the energy input and efforts in solving problematic stress situations was related to a greater extent with increasing the level of acculturation to organizational values and practices, than do preferences and choice of changing the situation or planning and organizing time. In relatively least extent, the organizational acculturation correlated with the Confidence in success coping strategy manifested in a positive interpretation of stressful events as useful and manageable. In fact, the all four coping strategies found as statistically significantly correlating with organizational acculturation are part of the active control-oriented coping, pointed out as the most effective approach to overcome stress. 
Table 1:Correlations between organizational acculturation and coping strategies

\begin{tabular}{|l|c|c|}
\hline \multirow{2}{*}{ Pearson's correlation (r) } & \multicolumn{2}{|c|}{ Organizational acculturation } \\
\cline { 2 - 3 } & $\mathbf{R}$ & $\mathbf{p}$ \\
\hline Increase of efforts & $.284^{* *}$ & .000 \\
\hline Escape from situation & & .010 \\
\hline Confidence in success & $.176^{*}$ & .004 \\
\hline Seeking social support & & .009 \\
\hline Change of situation & $.198^{* *}$ & \\
\hline Time management & $.178^{* *}$ & \\
\hline Reconciliation with situation & & \\
\hline
\end{tabular}

** The correlation is statistically significant at the level of $\mathrm{p}<0.01$.

* The correlation is statistically significant at the level of $p<0.05$.

In conclusion, the results obtained from the analysis of variance, verified, although partially, the first hypothesis raised in terms of the variables studied. The level of organizational acculturation depended only on part of the socio-demographic factors observed, including age, work experience in a particular organization and position in organizational hierarchy. Likewise, the frequency of use of four (out of seven) coping strategies was also influenced by a part of the socio-demographic factors observed, including age, marital status, level of education, total work experience and position in the organizational hierarchy.

The findinigs of correlation analysis implied that the increasing levels of organizational acculturation occurred together with the choice and use of control-oriented coping, highlighting the strategy of Increasing efforts as particularly the most important in that context. That gave grounds for a complete confirmation of the second hypothesis formulated.

These results can serve as a starting point for a future research on significant factors in determining the effectiveness of both acculturation and coping strategies in multicultural settings, and facilitate managers and employees to more effectively cope with cultural diversity and distance between individual and organizational values and practices.

\section{CONCLUSION}

The culturally diverse working environment implies greater possibility of increasing levels of acculturative and work stress which may be crucial to the overall wellbeing of individuals and a great challenge for contemporary organizations operating in a growing dynamic globalization and localization processes that put representatives of different cultures in continuous interaction of one another, even in the workplace. Thereby, it is essential that the individual and organizational implications of new forms of stress would be recognized and taken into account, so that to be found new ways for more effective coping with cultural diversity and cultural distance between individual and organizational values and practices. That, in turn, can lead to improvements not only in organizational performance, but also in employees' health and wellbeing in the increasingly difficult work conditions of today's workplace. 


\section{REFERENCES}

[1] Berry, J. (2001). A Psychology of Immigration. In: Journal of Social Issues, 57(3): 615-631.

[2] Berry, J. (2002). Cross-Cultural Psychology: Research and Applications. 2nd ed., Cambridge: Cambridge University Press.

[3] Berry, J. (2006a). Contexts of Acculturation. In: Sam, D. L. \& Berry, J. W. (Eds.), The Cambridge Handbook of Acculturation Psychology. Cambridge: Cambridge University Press, 27-42.

[4] Berry, J. (2006b). Stress Perspectives on Acculturation. In: Sam, D. L. \& Berry, J. W. (Eds.), The Cambridge Handbook of Acculturation Psychology. Cambridge: Cambridge University Press, 43-57.

[5] Berry, J. (2006c). Acculturative Stress. In: Wong, P. \& Wong, L. (Eds.), Handbook of Multicultural Perspectives on Stress and Coping. New York: Springer Science and Business Media, Inc., 287-298.

[6] Gailliard, B., Myers, K.,Seibold, D. (2010). Organizational Assimilation: A Multidimensional Reconceptualization and Measure. In: Management Communication Quarterly, 24: 552-578.

[7] Ilieva, S. (2008). Upravlenie na konfliktite y stresa v organizaciyata [Management of Conflict and Stress in the Organization]. In: Annual of Sofia University "St. Kliment Ohridski", vol. Psychology, 93: 127-145 (BULGARIAN).

[8] Latack, J, Havlovic, S. (1992). Coping with Job Stress: A Conceptual Evaluation Framework for Coping Measures. In: Journal of Organizational Behavior, 13(5): 479508.

[9] Palmer, S., Cooper, C., Thomas, K. (2004). “A Model of Work Stress to Underpin the Health and Safety Executive Advice for Tackling Work-related Stress and Stress Risk Assessments. Counselling at Work" <http://www.bacpworkplace.org.uk/ journal_pdf/acw_winter04_a.pdf>, (last access: 01/07/2016).

[10] Ward, C., Bochner, S., Furnham, A. (2003). The Psychology of Culture Shock. 2nd ed., New York: Routledge.

\section{Article history:}

- $\quad$ Received 1 November 2016

- Accepted 5 December 2016 